\title{
The Development of Digital Resources in Libraries and the Innovation of Strategies
}

\author{
Zhao Yanping \\ Xizang Minzu University, Shaanxi, Xianyang, 712000
}

Keywords: digital resource construction; information sharing; library; personalized service

\begin{abstract}
With the development of the times, libraries are also advancing with the times, constantly updating internal resources, and providing services to people through more scientific and convenient methods. Library digital resources have gradually become the focus of modern library development. Based on the digital resources construction of the library, this paper puts forward the strategy of library digital resources construction and hopes to provide some reference for the library digital construction.
\end{abstract}

\section{Introduction}

With the continuous development of the society and the science of our country, the development of digital management of libraries has gradually matured. In today's information society, the outstanding advantage of library digital resources is to provide readers with better services. Therefore, at this stage, many libraries have invested a lot of energy in the construction of digital resources, which in turn enables digital libraries to meet readers' requirements for study, work, research, and teaching.

\section{Library Digital Resources Construction}

\subsection{Digital Resources}

Digital resources are specifically a mixture of multiple types of information. The forms of existence are more diverse, including numbers, pictures, etc. Digital resources as a kind of information can be read by readers through computer networks. For readers, digital resources can not only help them obtain more comprehensive information resources, but also can run through the standards of various protocols, thereby achieving resource sharing and maximizing resource utilization. Digital resources are a relatively new way of obtaining information. This approach has greatly changed the development of the library. Library digital resources provide readers with more thoughtful and scientific quality services.

\subsection{Features of Digital Resource Construction}

(1) Comprehensiveness

According to related surveys, during the construction of library digital resources, the types of digital resources are very numerous and can cover all types of databases, specifically including resources such as periodicals, papers, patents, reference books, and annual inspections, among which are journal articles, The dissertation and e-books are the most cited. The most prominent is that full-text data resources have reached more than $90 \%$.

(2) Extensiveness

At this stage, the resources of the library can basically include all subjects, and the database of China Knowledge Network is analyzed. The results show that there are a large number of readers who navigate through subjects and categories. The major subjects include natural sciences, life sciences, and humanities. Mainly reflected in these categories of application and basic classes.

(3) Distribution

Digital resources as a kind of information, the characteristics of this information include a global 
distributed structure. Due to the distributed model and structure of digital resources, it is given asymmetry, disorder, and imbalance. It is precisely because of these characteristics of digital resources that it is possible not to be restricted by territoriality and thus to achieve a global distribution. The development of digital resources has a certain regionality. Specifically, in the more economically developed regions such as North China and East China, due to the more developed economy and more abundant funds, libraries in these regions have the ability to introduce digital resources. Obvious advantages, but relatively speaking, the number of library digital resources introduced in the economically backward areas is limited.

\section{Countermeasures for the Construction of Digital Resources in Libraries}

\subsection{Establishing a Professional Digital Library}

The library took one-sided consideration into its own needs in the process of digital resource development. It did not consider such factors as the efficiency of database usage and the frequency of citations. The digital resources construction of libraries should fully consider disciplines and professions, scientifically and rationally sum them up , and maximize the use of digital information resources through reasonable introduction. In this process, the unity of standards is very important. Promoting the development of digital information technology has a direct impact. At the same time, non-professional resource planning not only affects the efficiency of information utilization, but also makes the use of library digital resources more unfavorable. Therefore, the promotion of professionalism in the development and construction of digital resources in libraries has a very important role in enhancing efficiency and value.

\subsection{Building a Library Alliance to Strengthen Co-construction and Sharing of Resources}

The digital resources of the library can realize the simultaneous use, dissemination and reproduction of many people. The construction of digital resources makes the connection between the library and the library more compact and facilitates the co-construction and sharing of library resources. Libraries and libraries can establish alliances to minimize the construction cost of digital resources through joint procurement as much as possible, and then achieve low-cost co-construction and sharing of library resources, not only avoiding individual library resources. The phenomenon of insufficiency also solved the duplication of digital resources and wastage, improved the digital resource efficiency of libraries, and also promoted the common development of all libraries.Building library alliances further strengthens the sharing of digital resources and this can maximally mobilize the library's information resources. It is very important to enhance the protection capabilities of digital resources. In this way, the overall benefits of digital resources can be brought into play, which has played a significant role in solving the problem of insufficient resources for individual libraries. The way to establish a library union can use the resources advantages of other libraries to build professional information resources. On the basis of collaboration, we will complement each other with the advantages of digital resources and provide a vast space for content sharing.

The development and construction of library digital resources requires sufficient economic resources. Under normal circumstances, the funds for establishing a digital resource pool are in the range of hundreds of thousands. Due to the limitation of economic conditions, libraries often have problems in the process of digital resource construction, which seriously affects the development of digital resources. In addition, the construction of digital resources in libraries requires the purchase of comprehensive databases, which has led to serious professional limitations. Therefore, the emergence of a sound digital resource sharing platform is the key to realize the digital transformation and sharing network of the library. In this way, readers can truly realize resource information sharing through this platform.

\subsection{Promotion of personalized services}

For modern libraries, personalized services have gradually become a requirement for the 
development of digital resources. Library digital resource consultation can promote the normalization and standardization of human cost, service quality, and information. For library consulting services, achieving the unity of the three is the trend of future development. For the library information navigator, the development of the library's digital resources is characterized by individualized development and better information and consulting services based on the development of personalized services. Beyond the first time, in order to be able to provide individualized services to readers, management personnel should do a good job in the retrieval of data resources and maximize the value of library digital resources. The development of library digital resources cannot be separated from the needs of the demanders, library digital resources for readers to customize. This will not only enable the coordination of digital resources and human resources, but also achieve the ultimate goal of information sharing. Libraries should actively participate in the provision of personalized services, implement their own service consulting system as far as possible, meet the different requirements of readers, and then establish a standard system of modern library digital resources.

\subsection{Reasonable selection of digital resources that meet the actual library conditions}

According to the characteristics of the library and the actual situation as well as the funding situation of the library, a scientific and rational digital resource development and construction plan will be formulated. The establishment of a scientific and reasonable document digital resource development and construction plan can effectively ensure the balance and consistency of library digital data construction, and then promote the development and sharing of library digital document resources. For many libraries, it is difficult to build a complete range of digital resources due to the constraints of funding. Compared with the more professional documents, users may be more concerned with some theoretical knowledge that is more practical, so Chinese electronic Books and e-journals should be the first choice for library digital data construction at the present stage. Chinese e-books and e-journals are the key digital data. At the same time, image databases, video databases, and school-related databases should also be introduced as supporting materials.

\section{Conclusion}

In summary, the goal of the library to build a digital information sharing network is to share data and document resources. Therefore, the construction of digital resources in the library can effectively prevent unnecessary waste of resources, while also avoiding unnecessary maintenance and human resources cost. Beyond the first time, establishing an overall concept plays an important role in the construction and development of library digital resources, and actively promotes the digitalization of library resources in order to maximize the use of resources.

\section{References}

[1] Shen Lulu. Digital Library Resource Construction Strategy Based on "Intellectual Property"[J]. Science and Technology Innovation Report, 2013(6):228-229.

[2] Zhao Hongbo. The Big Data Environment Explores the Development Strategy of Digital Library Construction [J]. Science and Technology Innovation Report, 2017(11):252-253.

[3] Luan Rong. An Analysis of Status Quo and Construction Strategies of Digital Resources Utilization in Adult College Libraries [J]. Adult Education, 2016, 36(7):81-83.

[4] Zhang Xinglong. Study on the Strategies of Digital Resources Construction in Private University Libraries under the Background of MOOC [J]. Shanxi Science and Technology, 2017(2):70-72.

[5] Xu Jianye. The Digital Resources Construction of Library under the Background of the Development of Public Digital Culture [J]. New Century Library, 2015(11):57-59. 